\title{
On the Design of Nearly-PR and PR FIR Cosine Modulated Filter Banks Having Approximate Cosine-Rolloff Transition Band
}

\author{
S. S. Yin, S. C. Chan, Member, IEEE, and K. M. Tsui
}

\begin{abstract}
This paper proposes an efficient method for designing nearly perfect reconstruction (NPR) and perfect reconstruction (PR) cosine modulated filter banks (CMFBs) with prototype filters having an approximate cosine-rolloff (CR) transition band. It is shown that the flatness condition required for an NPR CMFB can be automatically satisfied by using a prototype filter with a CR transition band. The design problem is then formulated as a convex minimax optimization problem, and it can be solved by second-order cone programming (SOCP). By using the NPR CMFB so obtained as an initial guess to nonlinear optimizers such as Fmincon in Matlab, high-quality PR CMFBs can be obtained. The advantages of the proposed method are that it does not require a user-supplied initial guess of the prototype filter and bumps in the passband of the analysis filters can be effectively suppressed.
\end{abstract}

Index Terms-Cosine modulated filter banks (CMFBs), cosinerolloff (CR) transition band, nearly perfect reconstruction (NPR), perfect reconstruction (PR).

\section{INTRODUCTION}

$\mathbf{P}$ ERFECT reconstruction (PR) filter banks (FBs) have important applications in signal processing. A very efficient class of FBs is the cosine modulated FB (CMFB) [1], [2], which has very low design and implementation complexities and excellent frequency selectivity. The design of a PR or NPR system is usually obtained by iterative procedures involving nonlinear constrained optimization. When the number of variables and constraints increases, the optimization procedure is rather sensitive to the initial value of the prototype filter (PF), especially for CMFBs with low reconstruction delay. This has motivated considerable studies in the design and selection of effective initial guess for the PF in order to obtain faster convergence speed and better performance [3], [4]. Most design methods [5], [6] are focused on NPR CMFBs with linear-phase (LP) PFs. A multistep approach was proposed in [7] for designing NPR low-delay (LD) CMFBs starting with an FB with a smaller number of channels and shorter filter length. Recently, algorithms based on second-order cone programming (SOCP) were proposed in [8] for designing NPR and PR CMFBs.

In this paper, the design of NPR and PR CMFBs with PFs having an approximate cosine-rolloff $(\mathrm{CR})$ transition band is

Manuscript received June 9, 2006; revised March 20, 2007, June 14, 2007, and September 9, 2007. The work was presented in part at EUSIPCO, September 2004. This paper was recommended by Associate Editor Y.-P. Lin.

The authors are with the Department of Electrical and Electronic Engineering, The University of Hong Kong, Hong Kong (e-mail: ssyin@eee.hku.hk; scchan@eee.hku.hk; kmtsui@eee.hku.hk).

Digital Object Identifier 10.1109/TCSII.2007.916733 studied. The proposed CR PF is motivated by extending the formulation originally proposed in [1] and [2] for LP PF to the LD case. With the proposed PF, the condition for achieving a low-magnitude reconstruction error, which is the so-called magnitude flatness condition, required for NPR CMFBs can be approximately satisfied. The design problem can be formulated as a convex minimax or least-square (LS) optimization problem. Therefore, its optimal solution can be solved by the Parks-McClellan algorithm, SOCP, or semidefinite programming (SDP) [9], [10]. Compared with PR systems, NPR systems have a larger stopband attenuation at the expense of a higher reconstruction error. Another advantage of NPR CMFBs is their low design complexity, because the PR constraints are only approximately satisfied. Design results show that the reconstruction error of the NPR CMFBs obtained by the proposed method is reasonably low. Moreover, it is found that the CR condition can significantly suppress the bumping in the first and last analysis filters of the FBs, which are commonly encountered in conventional methods for designing LD CMFBs [8], [11]. Furthermore, by using the NPR CMFBs designed as the initial guesses to constrained optimization software, such as the Fmincon function in the Matlab OPTIM toolbox, high-quality PR CMFBs can readily be obtained. Since Fmincon is based on sequential quadratic programming (SQP), which represents the state of the art in nonlinear programming solvers, the quality of the solution found will be similar to the SOCP-based method in [8]. On the other hand, as the method in [8] is specifically designed for the given problem, it potentially requires a lower computational time. Nevertheless, a proper initial guess is very important for both methods in order to obtain good solutions.

This paper is organized as follows. In Section II, the principle of NPR CMFBs with the proposed CR PFs is described. Section III is devoted to the design of these CR PFs using SOCP. Several design examples and comparisons with other conventional methods are given in Section IV. Finally, a conclusion is drawn in Section V.

\section{NPR CMFBs WITH CR PFs}

The frequency response of the PF $P(z)$ for a CMFB can be written as $P\left(e^{j \omega}\right)=P_{M}\left(e^{j \omega}\right) e^{-j \omega D / 2}$, where $D / 2$ is the desired group delay of the filter. For LP PFs, $P_{M}\left(e^{j \omega}\right)$ is the magnitude response of the filter and it is a real function, while, for its LD counterpart, it is a complex function approximating the magnitude response of the filter. For an $M$-channel maximally decimated NPR CMFB, the analysis filters are obtained by modulating the PF: $H_{k}(z)=a_{k} U_{k}(z)+a_{k}^{*} V_{k}(z)$, where $U_{k}(z)=c_{k} P\left(z W^{k+0.5}\right), V_{k}(z)=c_{k}^{*} P\left(z W^{-(k+0.5)}\right), a_{k}$, and 
$c_{k}$ are unit-magnitude complex constants and $W^{k}=W_{2 M}^{k}$, $=e^{-2 j k \pi /(2 M)}$, for $0 \leq k \leq M-1$ [1], [2]. The cutoff frequency of the PF, $\omega_{c}$, is $\bar{\pi} /(2 \bar{M})$. The main aliasing components in the reconstruction can be cancelled, if $a_{k}$ and $c_{k}$ are chosen as $a_{k}=e^{j \theta_{k}}, \theta_{k}=(-1)^{k}(\pi / 4)$, and $c_{k}=W^{(k+0.5) D / 2}$. The analysis and synthesis filters are then given by

$$
\begin{aligned}
& h_{k}(n)=2 p(n) \cos \left[\frac{\pi}{M}\left(k+\frac{1}{2}\right)\left(n-\frac{D}{2}\right)+(-1)^{k} \frac{\pi}{4}\right], \\
& f_{k}(n)=2 p(n) \cos \left[\frac{\pi}{M}\left(k+\frac{1}{2}\right)\left(n-\frac{D}{2}\right)-(-1)^{k} \frac{\pi}{4}\right]
\end{aligned}
$$

for $k=0, \ldots, M-1$ and $n=0, \ldots, N-1 .\{p(n)\}$ and $N$ are the impulse response and filter length of the PF and $D$ is the system delay. If $D<(N-1)$, it is an LD CMFB. The system transfer function is $T_{0}\left(e^{j \omega}\right) \approx(1 / M) \sum_{k=0}^{M-1}\left[U_{k}^{2}\left(e^{j \omega}\right)+V_{k}^{2}\left(e^{j \omega}\right)\right]$.

The magnitude distortion of the reconstruction can be minimized by making $\left|T_{0}\left(e^{j \omega}\right)\right|$ as close to a constant as possible. Supposing that the passband and stopband ripples are sufficiently small, then $\left|T_{0}\left(e^{j \omega}\right)\right|$ will be nearly constant in the passband of the analysis filters. Therefore, we need only to consider their transition bands. As $U_{k}\left(e^{j \omega}\right)$ and $V_{k}\left(e^{j \omega}\right)$ are frequency-shifted versions of $P\left(e^{j \omega}\right)$, we only need to impose the following constraint:

$$
\left|P\left(e^{j \omega}\right)\right|^{2}+\left|P\left(e^{j(\omega-(\pi / M)}\right)\right|^{2}=1
$$

where $\omega \in\left[0<\omega_{p}, \omega_{s}<\pi / M\right]$, and $\omega_{p}$ and $\omega_{s}$ are respectively the passband and stopband cutoff frequencies of $P\left(e^{j \omega}\right)$. Using the LS design criterion, the design problem is

$$
\min _{p}\left(\lambda \phi_{1}+(1-\lambda) \phi_{2}\right)
$$

where $\boldsymbol{p}=[p(0), \ldots, p(N-1)]^{T}$ is the variable vector, $\phi_{1}=\int_{\omega_{s}}^{\pi}\left|P\left(e^{j \omega}\right)\right|^{2} d \omega$ measures the stopband attenuation of the analysis filters, $\omega_{s}=(\pi /(2 M))+\varepsilon$ is the stopband cutoff frequency for some positive real number $\varepsilon$, $\phi_{2}=\int_{\omega_{p}}^{\omega_{s}}\left\{\left|P\left(e^{j \omega}\right)\right|^{2}+\left|P\left(e^{j(\omega-(\pi / M))}\right)\right|^{2}-1\right\}^{2} d \omega$ is the flatness measure which minimizes the magnitude reconstruction error, $\omega_{p}$ is the passband cutoff frequency, and $\lambda$ is a positive weighting factor providing a tradeoff between $\phi_{1}$ and $\phi_{2}$. Since $\phi_{2}$ is not quadratic, (3) has to be solved using unconstrained nonlinear programming. In [6], the NPR CMFB is designed by minimizing $\phi=\max \left\{\left|P\left(e^{j \omega}\right)\right|^{2}+\left|P\left(e^{j(\omega-(\pi / M))}\right)\right|^{2}-1\right\}$ using a modified Remez exchange method. More precisely, the passband cutoff frequency of the PF is iteratively adjusted so that the equi-ripple filters obtained from the Remez algorithm minimize the reconstruction errors. We now formulate the design problem as a convex optimization problem, which can be solved using SOCP or other appropriate methods. Assume that the desired frequency response of the PF is

$$
P_{d}\left(e^{j \omega}\right)= \begin{cases}e^{-j \omega D / 2}, & \omega \in\left[0, \omega_{p}\right] \\ \cos \left(\frac{\pi}{2 \Delta \omega}\left(\omega-\omega_{p}\right)\right) \cdot e^{-j \omega D / 2}, & \omega \in\left[\omega_{p}, \omega_{s}\right] \\ 0, & \omega \in\left[\omega_{s}, \pi\right]\end{cases}
$$

where the group delay is $D / 2$ over the passband and the transition band and $\Delta \omega=\omega_{s}-\omega_{p}$ is the transition bandwidth. We call this class of filters the CR low-pass filters because their transition bands follow a CR characteristic. The desired response in $\omega \in[-\pi, 0]$ is $P_{d}\left(e^{-j \omega}\right)$. Given the proposed CR PF, the flatness condition in (2) is automatically satisfied because

$$
\begin{aligned}
\left|P\left(e^{j \omega}\right)\right|^{2}+\left|P\left(e^{j(\omega-\pi / M)}\right)\right|^{2}= & \cos ^{2}\left(\frac{\pi}{2 \Delta \omega}\left(\omega-\omega_{p}\right)\right) \\
& +\sin ^{2}\left(\frac{\pi}{2 \Delta \omega}\left(\omega-\omega_{p}\right)\right)
\end{aligned}
$$

for $\omega \in\left[\omega_{p}, \omega_{s}\right]$. Since the flatness condition can be structurally imposed, the design problem is reduced to the design of a lowpass FIR filter with the CR transition band given by (4). We shall see from design examples to be presented that this is very effective in suppressing bumping in the passband of the analysis filters which is commonly found in the conventional methods.

\section{DESIGN METHOd UsING SOCP}

As mentioned earlier, the design problem can be performed by LS and Parks-McClellan algorithms and SOCP and SDP, depending on the objective functions and constraints imposed. Due to the generality and good performance of SOCP, we shall formulate our problem using this framework. Without loss of generality, we shall consider the difficult design problem for the minimax criterion as follows:

$$
\begin{aligned}
& \text { Minimize } \delta \\
& \text { Subject to } W(\omega)\left|P\left(e^{j \omega}\right)-P_{d}\left(e^{j \omega}\right)\right| \leq \delta
\end{aligned}
$$

where $-\pi \leq \omega \leq \pi, \delta$ is an additional variable for the maximum ripple, and $W(\omega)$ is a positive weighting function. To simplify notation, the frequency response of the prototype is denoted by $P(\omega)=\sum_{n=0}^{N-1} p(n) e^{-j n \omega}=\boldsymbol{p}^{T}\{c(\omega)-j s(\omega)\}$, where $\boldsymbol{p}$ is the variable vector, $c(\omega)=[1, \cos (\omega), \ldots, \cos ((N-$ 1) $\omega)]^{T}$, and $s(\omega)=[1, \sin (\omega), \ldots, \sin ((N-1) \omega)]^{T}$. Let $P_{R_{-} d}(\omega)$ and $P_{I_{-} d}(\omega)$ be the real and imaginary parts of $P_{d}\left(e^{j \omega}\right)$, and then (5) can be further rewritten as

$$
\begin{gathered}
\text { Minimize } \delta \\
\text { Subject to } \delta-\left\{a_{R}^{2}(\omega)+a_{I}^{2}(\omega)\right\}^{1 / 2} \geq 0
\end{gathered}
$$

where $-\pi \leq \omega \leq \pi, a_{R}(\omega)=W(\omega)\left[\boldsymbol{p}^{T} \boldsymbol{c}(\omega)-P_{R \_d}(\omega)\right]$, and $a_{I}(\omega)=\bar{W}(\omega)\left[\boldsymbol{p}^{T} \boldsymbol{s}(\omega)+P_{I_{-} d}(\omega)\right]$. Discretizing the frequency variable $\omega$ over a dense set of frequencies $\left\{\omega_{i}, 1 \leq i \leq m\right\}$ on the frequency interval of interest, one obtains the following standard SOCP problem:

$$
\begin{aligned}
& \text { Minimize } \boldsymbol{c}^{T} \boldsymbol{x} \\
& \text { Subject to } \quad \boldsymbol{c}^{T} \boldsymbol{x} \geq\left\|\boldsymbol{F}_{i} \boldsymbol{x}-\boldsymbol{g}_{i}\right\|_{2}
\end{aligned}
$$

where $\boldsymbol{g}_{i}=W\left(\omega_{i}\right)\left[-P_{R_{-} d}\left(\omega_{i}\right) \quad P_{I_{-} d}\left(\omega_{i}\right)\right]^{T}, \boldsymbol{c}=\left[1, \boldsymbol{O}_{N}^{T}\right]^{T}$, $\boldsymbol{x}=\left[\delta, \boldsymbol{p}^{T}\right]^{T}$, and

$$
\boldsymbol{F}_{i}=W\left(\omega_{i}\right)\left[\begin{array}{ll}
0 & \boldsymbol{c}\left(\omega_{i}\right)^{T} \\
0 & \boldsymbol{s}\left(\omega_{i}\right)^{T}
\end{array}\right]
$$


TABLE I

DESIGN PROCEDURE

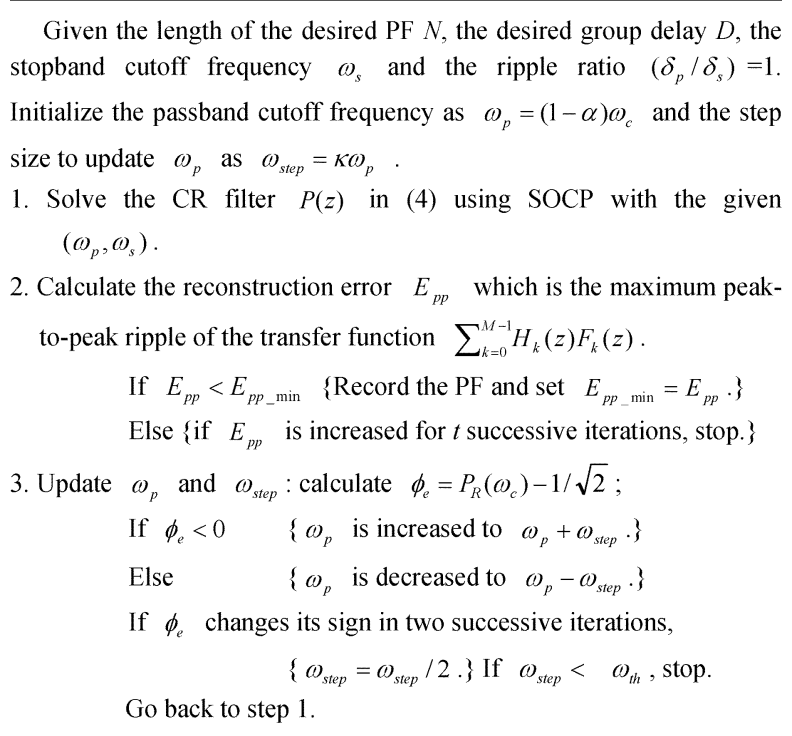

$\boldsymbol{O}_{N}$ is an $N$-row zero vector and $\|\cdot\|_{2}$ denotes the Euclidean norm. Because it is a convex optimization problem, the optimality of the solution is guaranteed, if it exists.

Since NPR CMFBs so obtained are of very high quality, they can serve as an initial guesses to the constrained nonlinear optimizer to solve for the PR CMFB with the same filter parameters. Due to page limitations, the formulation is omitted here. Interested readers are referred to [1] for details. In this work, we use the function Fmincon in Matlab. The termination tolerance on the constraint value and the maximum number of iterations allowed for Fmincon are set to 1e-15 and 1000, respectively.

\section{Design Procedure AND EXAMPLES}

It is observed that there is a tradeoff among the transition bandwidth, the ratio of passband and stopband ripples of the $\mathrm{PFs}$, and the reconstruction error during the design of these NPR CMFBs. Generally speaking, it is possible to improve the performance of the CR NPR CMFB further by optimizing the transition bandwidth and ripples of the CMFB. Next, a design procedure based on SOCP is presented.

The low-pass CR filter in (4) can be designed by using SOCP with an additional amplitude constraint imposed at $\omega_{c}$. Moreover, for a given ratio of passband and stopband ripples of the $\mathrm{PF}$, the passband cutoff frequency $\omega_{p}$ can be adjusted iteratively based on the error at $\omega_{c}$, while the stopband cutoff frequency $\omega_{s}$ remains fixed to minimize the reconstruction error. Instead of imposing the constraint on the entire transition band in (4), only a single constraint at $\omega_{c}$ is imposed to avoid constraining excessively the PFs. Let $\phi_{e}=P_{R}\left(\omega_{c}\right)-1 / \sqrt{2}$ be the CR error at $\omega_{c}$. To satisfy the amplitude constraint at $\omega_{c}$, we assume $\phi_{e}$ is approximately convex so that we can solve for it using a bisection-like search. Basically, for a given $\left(\omega_{p}, \omega_{s}\right)$, the problem in (7) is solved using SOCP. If the corresponding $\phi_{e}$ is greater than zero, $\omega_{p}$ is decreased. Otherwise, it is increased. The algorithm stops if the $E_{\mathrm{pp}}$ is increased in $t$ successive iterations. The $\omega_{p}$ giving the minimum $E_{\mathrm{pp}}$ will be recorded. The design procedure is summarized in Table $\mathrm{I}$.
TABLE II

EIght-Channel NPR CMFBs With DifFERENT Delays $(\alpha, \kappa)=(0.7,0)$

\begin{tabular}{ccccccc}
\hline \hline FB & $D$ & $\omega_{p}$ & $A_{s}(\mathrm{~dB})$ & $A_{e}(\mathrm{~dB})$ & $E_{p p}$ & $\mathrm{DT}(\mathrm{s})$ \\
\hline F-1 & 95 & $0.01875 \pi$ & -98.04 & -114.46 & $7.64 \mathrm{e}-3$ & 5.79 \\
$\mathrm{~F}-2$ & 111 & $0.01875 \pi$ & -103.42 & -117.78 & $1.48 \mathrm{e}-3$ & 6.76 \\
F-3 & 127 & $0.01875 \pi$ & -112.60 & -125.79 & $1.59 \mathrm{e}-3$ & 6.40 \\
F-4* & 127 & $0.017103243 \pi$ & -112.19 & -125.10 & $2.03 \mathrm{e}-3$ & 2.90 \\
\hline \multicolumn{7}{l}{ * designed using method in [6]. }
\end{tabular}

TABLE III

EIGHT-ChANNEL NPR CMFBs With DiFFERENT Delays $(\alpha, \kappa)=(0.4,0.6)$

\begin{tabular}{ccccccc}
\hline \hline FB & $D$ & $\omega_{p}$ & $A_{s}(\mathrm{~dB})$ & $A_{e}(\mathrm{~dB})$ & $E_{p p}$ & $\mathrm{DT}(\mathrm{s})$ \\
\hline $\mathrm{F}-1^{\prime}$ & 95 & $0.0184375 \pi$ & -98.66 & -115.67 & $8.96 \mathrm{e}-3$ & 75.73 \\
$\mathrm{~F}^{\prime}{ }^{\prime}$ & 111 & $0.0184375 \pi$ & -105.32 & -117.90 & $1.48 \mathrm{e}-3$ & 76.46 \\
$\mathrm{~F}^{\prime}{ }^{\prime}$ & 127 & $0.0203125 \pi$ & -113.00 & -126.00 & $1.58 \mathrm{e}-3$ & 81.56 \\
\hline
\end{tabular}

$\alpha$ and $\kappa$ are constant real numbers both less than 1 to initialize $\omega_{p}$ and $\omega_{\text {step }}$. Experimental results showed that a satisfactory solution can be found even when $\alpha$ and $\kappa$ are chosen as 0.7 and zero (i.e., no bisection-like search) with $\omega_{s}=\pi / M$. A better solution may be obtained by iteratively adjusting $\omega_{p}$ and $\omega_{\text {step }}$ based on $\phi_{e}$ using the bisection-like search. If $E_{\mathrm{pp}}$ does not decrease in $t$ (such as $t=5$ in our cases) successive times, we assume the local solution is found. The algorithm also stops if $\omega_{\text {step }}$ is smaller than $\omega_{\text {th }}$, a constant such as 1e-7. Though we can adjust the stopband instead of the passband edges, a large aliasing error will result if $\omega_{s}$ is increased beyond $\pi / M$. Therefore, we prefer to vary $\omega_{p}$. Moreover, an optional outer loop, in which the ripple ratio $\left(\delta_{p} / \delta_{s}\right)$ is also adjusted, can be incorporated into the proposed procedure if required.

A similar CR PF was also considered in [12] for the LP case. It differs from (4) in that the whole passband and stopband is obtained by transforming a cosine function by a polynomial. It is rather difficult to define the cutoff frequencies and ripples of the various bands. Moreover, it uses the LS criterion, and the problem is solved using the eigenfilter approach. To solve for a PR CMFB with the same filter parameters, we can minimize

$$
\phi(\boldsymbol{p})=\lambda \cdot \int_{\omega_{s}}^{\pi}\left|P\left(e^{j \omega}\right)\right|^{\rho} d \omega+(1-\lambda) \cdot \int_{0}^{\omega_{p 0}}|| H_{0}\left(e^{j \omega}\right)|-1|^{\rho} d \omega
$$

subject to the PR constraints, where $\boldsymbol{p}$ is the variable vector which is the same as in (3). The first part measures the stopband attenuation of the PF and the second part is the passband flatness measure of the first analysis filter obtained $\left(\omega_{p 0}\right.$ denotes the passband cutoff frequency of the first analysis filter). $\lambda$ is a positive weighting factor providing a tradeoff between the two parts. For CMFBs with an LP PF, $\lambda$ is set to 0 . Common choices of $\rho$ are $\rho=1$ or $\rho=2$ (the LS criterion).

\section{A. Example 1: Eight-Channel NPR CMFBs With Different Delays}

To illustrate the efficiency of the proposed method, several eight-channel CR NPR CMFBs with different delays are designed as summarized in Table II. The filter length $N$ of all the FBs is 128. $D$ is the system delay, $A_{s}(\mathrm{~dB})$ is the lowest stopband attenuation of the $\mathrm{PF}$, $A_{e}=\max _{1 \leq l \leq M-1} \mid T_{l}\left(e^{j \omega)} \mid(\mathrm{dB})\right.$ is the worst-case aliasing error, 


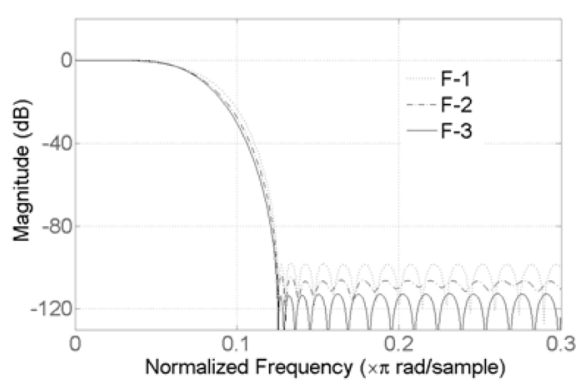

(a)

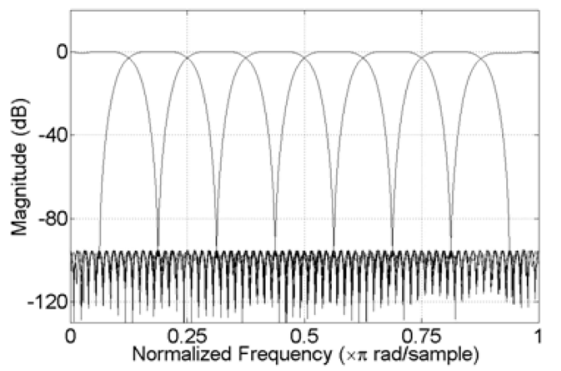

(b)

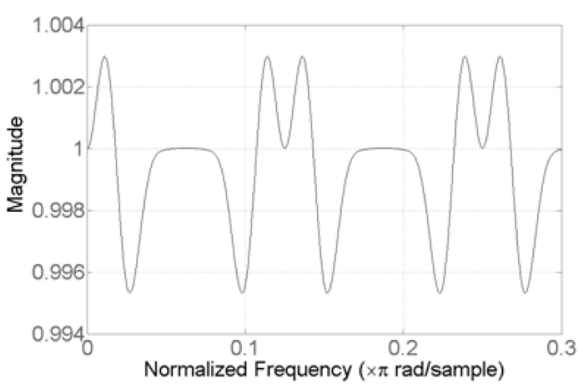

(c)

Fig. 1. (a) The CR PFs of FBs in Table II. (b) Analysis filters and (c) reconstruction error of LD CMFB: F-1 ( $D=95)$ in Table II.

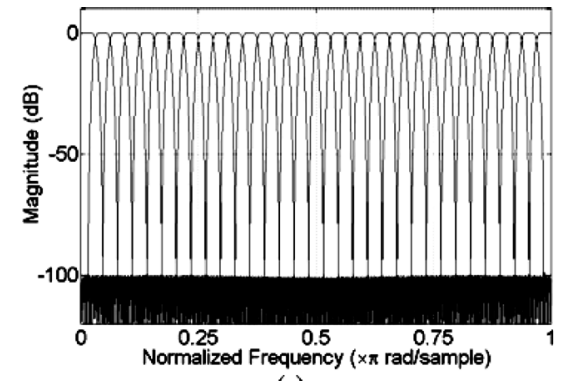

(a)

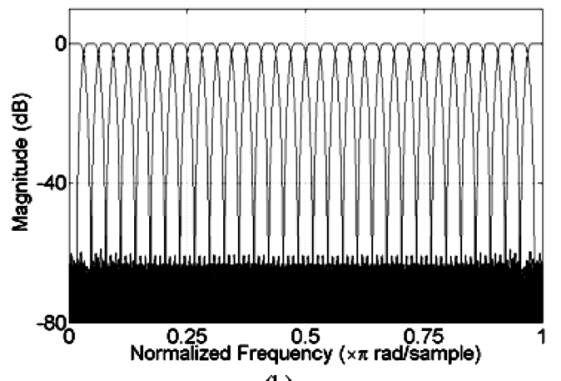

(b)

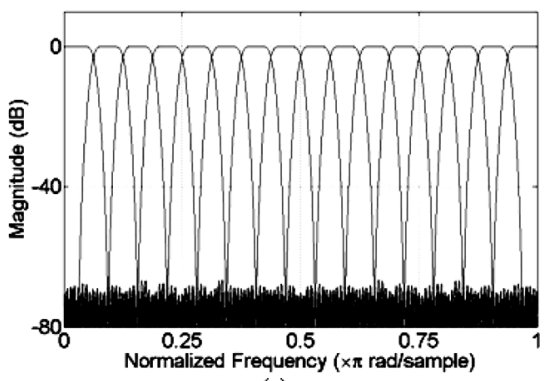

(c)

Fig. 2. Analysis filters of (a) NPR32 CMFB in Table IV, (b) PR32-S CMFB, and (c) PR16-S CMFB in Table V.

TABLE IV

16/32-CHANNEL NPR CMFBs $\left\{(\alpha, \kappa)=(0.7,0)\right.$ and $\left.\omega_{s}=\pi / M\right\}$

\begin{tabular}{ccccccc}
\hline \hline FB & $M$ & $N$ & $A_{s}(\mathrm{~dB})$ & $A_{e}(\mathrm{~dB})$ & $E_{p p}$ & DT(s) \\
\hline NPR16 & 16 & 384 & -168.95 & -192.75 & $3.27 \mathrm{e}-3$ & 68.4 \\
NPR32 & 32 & 448 & -101.94 & -125.76 & $2.38 \mathrm{e}-3$ & 90.4 \\
\hline
\end{tabular}

where $T_{l}\left(e^{j \omega)} \mid=1 / M \sum_{k=0}^{M-1} F_{k}\left(e^{j \omega}\right) H_{k}\left(e^{j(\omega-2 \pi l / M)}\right)\right.$ for $1 \leq l \leq M-1$, and $E_{\mathrm{pp}}$ is the maximum peak-to-peak ripple of the transfer function. The design procedure was applied with $\alpha$ and $\kappa$ chosen as $(0.7,0)$, while $\omega_{s}=\pi / M$ for all cases designed. The design time (DT), using SeDuMi 1.05R5 toolbox with Matlab version 7.0, in a Pentium(R) 4 CPU 3.00 $\mathrm{GHz} \mathrm{PC}$, is also listed in Table II. Comparing the eight-channel NPR CMFB with the one using the LP PF designed in [6] (F-4 in Table II), the proposed method (F-3 in Table II) gives a lower reconstruction error, a higher stopband attenuation, and a narrower transition band. This is because the proposed method further exploits the tradeoff among the transition width, ratio of passband and stopband ripples, and the reconstruction error. The bisection-like search version of the proposed procedure was also studied with $\alpha$ and $\kappa$ chosen as $(0.4,0.6)$, while the other designed parameters were the same as in Table II. The designed CMFBs (F-1'-F-3') are listed in Table III. It can be seen that a better solution may be found using the bisection-like search method at the cost of a longer design time.

The magnitude responses of the CR PFs in Table II are plotted in Fig. 1(a). The magnitude response and reconstruction error of the LD NPR CMFB with $D=95$ are plotted respectively in Fig. 1(b) and (c).

\section{B. Example 2: 16/32-Channel NPR/PR CMFBs With LP PFs}

Two NPR CMFBs with CR LP PFs, designed using the proposed method, are summarized in Table IV. Comparing with
TABLE V 16/32-CHANNEL PR CMFBS

\begin{tabular}{cccccc}
\hline \hline FB & $M$ & $N$ & $A_{s}(\mathrm{~dB})$ & $E_{p p}$ & Iters \\
\hline PR32-S & 32 & 448 & -60.47 & $9.86 \mathrm{e}-13$ & 226 \\
PR32-R & 32 & 448 & -60.35 & $4.84 \mathrm{e}-14$ & 276 \\
PR32-W & 32 & 448 & -50.07 & $3.64 \mathrm{e}-13$ & 359 \\
PR16-S & 16 & 384 & -62.62 & $1.72 \mathrm{e}-13$ & 248 \\
PR16-R & 16 & 384 & -49.01 & $5.66 \mathrm{e}-14$ & 459 \\
PR16-W & 16 & 384 & -50.68 & $1.66 \mathrm{e}-13$ & 743 \\
PR16 [8] & 16 & 384 & -100 & $1.81 \mathrm{e}-7$ & 147 \\
\hline
\end{tabular}

the NPR CMFB designed in [8], the proposed method offers a comparable performance, but an initial guess of the $\mathrm{PF}$ is not required. Fig. 2(a) shows the frequency response of one CMFB, NPR32 in Table IV, which has a comparable performance to the 32-channel NPR CMFB presented in [8, Example 1].

Both NPR CMFBs designed above are then used as initial guesses to obtain PR CMFBs using the measure in (8) with $\rho=2$. The obtained CMFBs (PR16-S and PR32-S) are shown in Table $\mathrm{V}$ and Fig. 2(b) and (c). Iters denotes the number of iterations for obtaining the PR CMFB by Fmincon in the proposed method. For comparison purposes, different initial guesses with the same filter parameters designed using Remez and Window-method (Hamming) were used to solve for PR CMFBs (PRM-R or PRM-W in Table V). It was found that the proposed method lead to a PR CMFB with an improved stopband attenuation $(-60.47 \mathrm{~dB}$ versus $-60.35 \mathrm{~dB}$ or $-50.07 \mathrm{~dB}$; $-62.62 \mathrm{~dB}$ versus $-49.01 \mathrm{~dB}$ or $-50.68 \mathrm{~dB}$ ) and a faster convergence speed in terms of the number of iterations (226 versus 276 or $359 ; 248$ versus 459 or 743 ) than using a conventional LP filter as an initial guess. Compared with a similar 16-channel PR CMFB presented in [8] (also listed in Table V), 


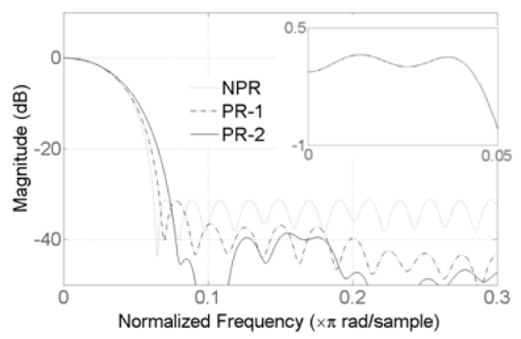

(a)

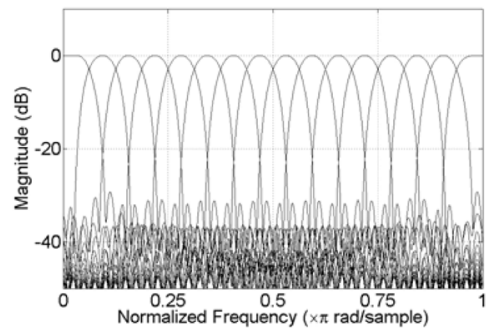

(b)

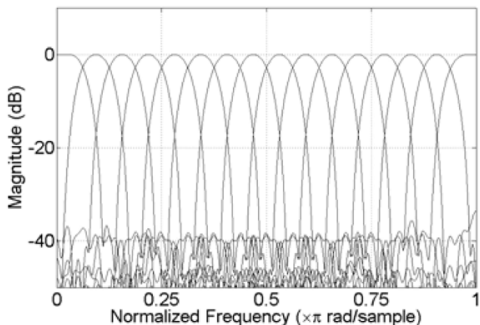

(c)

Fig. 3. (a) The CR PFs of FBs in Table VI (the smaller figure shows the passband detail of the first analysis filter of the NPR LD CMFB). (b) Analysis filters of PR-1 CMFB and (c) analysis filters of PR-2 CMFB in Table VI.

TABLE VI

16-CHANNEL NPR/PR CMFBs WITH LD PFS

\begin{tabular}{ccccccc}
\hline \hline FB & $N$ & $D$ & $A_{s}(\mathrm{~dB})$ & $E_{p p}$ & Iters & DT(s) \\
\hline NPR & 96 & 63 & -31.22 & $5.71 \mathrm{e}-2$ & 19 & 2.9 \\
PR-1 $(\rho=2)$ & 96 & 63 & -31.52 & $4.28 \mathrm{e}-14$ & 13 & 7.28 \\
PR-2 $(\rho=1)$ & 96 & 63 & -38.64 & $1.50 \mathrm{e}-13$ & 281 & 130.72 \\
\hline
\end{tabular}

the proposed PR16-S offers a different design tradeoff, having a lower stopband attenuation $(-62.62 \mathrm{~dB}$ versus $-100 \mathrm{~dB})$ but a lower reconstruction error (1.72e-13 versus $1.81 \mathrm{e}-07)$. Since the proposed algorithm is different from that in [8], we do not compare directly the number of iterations with that in [8], though the latter was reported to be 147 .

\section{Example 3: 16-Channel NPR/PR CMFBs With LD PFs}

A 16-channel NPR CMFB with length 96 and a system delay of 63 samples was first designed using the proposed method. $\alpha$ and $\kappa$ are chosen as $(0.7,0)$, while $\omega_{s}=\pi / M$ and the ripple ratio $\left(\delta_{p} / \delta_{s}\right)$ is set to 5 . The design time is $2.9 \mathrm{~s}$ (19 iterations) and the resulted CMFB is illustrated in Fig. 3(a) and Table VI. Since the flatness condition is approximately satisfied by the CR PF, the NPR LD CMFB designed has a good frequency characteristic in the passband of the analysis filters, as shown in the smaller figure in Fig. 3(a).

The designed 16-channel NPR CMFBs were used as the initial guess for designing PR CMFBs using the measure in (8) with $\rho=2$ and $\rho=1$. The results are illustrated in Table VI and Fig. 3(b) and (c). The LS measure leads to a faster convergence speed but a lower stopband attenuation. A 16-channel PR CMFB with $N=96, D=63$ was designed using the quadratic-constrained least-square (QCLS) technique presented in [11]. Since only the stopband energy of the LD PF was formulated into the design problem, there are bumps in the passband of the analysis filters, which were also found in the design method in [8, Example 3]. On the other hand, the passband flatness of the analysis filter is incorporated into the proposed design method. Therefore, the passband of the analysis filters so obtained has a good frequency characteristic at the expense of a lower stopband attenuation.

A similar 128-channel LD NPR CMFB with $N=1792, D=$ 1279 was also designed using the proposed method and then further optimized to obtain a PR CMFB with $A_{s}=-39.21 \mathrm{~dB}$ and $E_{\mathrm{pp}}=3.16 \mathrm{e}-12$. A value of 467 iterations are needed for Fmincon to converge to the PR CMFB using the LS criterion. This shows the effectiveness of the proposed method in designing LD PR CMFBs with a high number of channels and long filter lengths.

\section{CONCLUSION}

An efficient method for designing NPR and PR CMFBs using PFs with an approximate CR transition band is presented. A design procedure based on SOCP is also developed. Design results show that the proposed method yields comparable or better performance than conventional methods, and it is able to suppress the undesirable bumps in the passband of the analysis filters.

\section{REFERENCES}

[1] P. P. Vaidyanathan, Multirate Systems and Filter Banks. Englewood Cliffs, NJ: Prentice-Hall, c1993.

[2] R. D. Koilpillai and P. P. Vaidyanathan, "A spectral factorization approach to pseudo-QMF design," IEEE Trans. Signal Process., vol. 41, no. 1, pp. 82-92, Jan. 1993.

[3] Y. P. Lin and P. P. Vaidyanathan, "A kaiser window approach for the design of prototype filters of cosine modulated filterbanks," IEEE Signal Process. Lett., vol. 5, no. 6, pp. 132-134, Jun. 1998.

[4] F. Cruz-Roldan, P. Amo-Lopez, S. Maldonado-Bascon, and S. S. Lawson, "An efficient and simple method for designing prototype filters for cosine-modulated pseudo-QMF banks," IEEE Signal Process. Lett., vol. 9, no. 1, pp. 29-31, Jan. 2002.

[5] T. Q. Nguyen, "Near-perfect-reconstruction pseudo-QMF banks," IEEE Trans. Signal Process., vol. 42, no. 1, pp. 65-76, Jan. 1994.

[6] S. K. Mitra, Digital Signal Processing: A Computer-Based Approach. New York: McGraw-Hill, 2001.

[7] R. Bregovic and T. Saramaki, "An efficient approach for designing nearly perfect-reconstruction low-delay cosine-modulated filter banks," in Proc. IEEE ISCAS, May 2002, vol. 1, pp. I-825-I-828.

[8] W. S. Lu, T. Saramaki, and R. Bregovic, "Design of practically perfect-reconstruction cosine-modulated filter banks: A second-order cone programming approach," IEEE Trans. Circuits Syst. I, Reg. Papers, vol. 51, no. 3, pp. 552-563, Mar. 2004

[9] W. S. Lu, "Design of nonlinear-phase FIR digital filters: A semidefinite programming approach," in Proc. IEEE ISCAS, May 1999, vol. 3, pp. 263-266.

[10] S. C. Chan and S. S. Yin, "On the design of low delay nearly-PR and PR FIR cosine modulated filter banks having approximate cosine-rolloff transition band," in Proc. EUSIPCO, Sep. 2004, pp. 1253-1256.

[11] P. N. Heller, T. Karp, and T. Q. Nguyen, "A general formulation of modulated filter banks," IEEE Trans. Signal Process., vol. 47, no. 4, pp. 986-1002, Apr. 1999.

[12] F. Argenti and E. Del Re, "Eigenfilter design of real and complex coefficient QMF prototype," IEEE Trans. Circuits Syst. II, Analog Digit. Signal Process., vol. 47, no. 8, pp. 787-792, Aug. 2000. 